\title{
Point-vortex model for Lagrangian intermittency in turbulence
}

\author{
Mark Peter Rast \\ Laboratory for Atmospheric and Space Physics and Department of Astrophysical and Planetary Sciences, University of Colorado, \\ Boulder, Colorado 80309, USA \\ Jean-Francois Pinton \\ Laboratoire de Physique de l'École Normale Supérieure de Lyon, CNRS UMR5672, 46 allée d'Italie, F-69364 Lyon, France
}

(Received 20 August 2008; published 14 April 2009)

\begin{abstract}
Experiments have shown that Lagrangian statistics in turbulent flows display Gaussianly distributed velocity values and non-Gaussianly distributed velocity differences or accelerations. Coherent flow structures in the form of vortices have often been proposed to play an important role in this behavior. Here we examine the origin of these statistics using both continuously stirred $n$-body point-vortex simulations and analytic random variable transformation in a simplified model of randomly distributed vortices. We conclude that Lagrangian velocity distributions can be understood in terms of dominant nearest vortex neighbor contributions. Accelerations likewise reflect vortical contributions, but at smallest temporal increment are dominated, not by the motion of the Lagrangian tracers, but by vorticity reconfiguration within the domain.
\end{abstract}

DOI: 10.1103/PhysRevE.79.046314

PACS number(s): 47.27.-i, 47.32.-y

\section{INTRODUCTION}

The analysis of the statistical properties of turbulence at small scales has shown that, while the energy distribution is well described by Kolmogorov's 1941 theory [1], velocity increments display strongly non-Gaussian distributions [2]. This behavior, usually called "intermittency" in turbulence studies, is firmly established via laboratory and numerical experiments for both Eulerian and Lagrangian [3-5] velocities. Analytical studies of simplified models have also shown that it is consistent with the structure of the Navier-Stokes equations [6], and in multifractal descriptions, Eulerian and Lagrangian intermittencies are related through the spatial granularity of energy dissipation [7-9]. One of the advantages of the Lagrangian description is that it casts intermittency in the dynamical framework of fluid particle motions. In this context, it has been proposed that the very high acceleration values measured originate in the proximity of strong vorticity filaments [3-5,10-13]. In the work reported here, we study this proposition directly by examining the motion of Lagrangian tracer particles in the flow field generated by the superposition of point-vortex contributions. Although it is clear that replacing a turbulent flow by a collection of point vortices is an idealized oversimplification $[2,14,15]$, the procedure sheds light on the role that vortex dynamics plays in the statistics of Lagrangian motions [13].

This paper presents the results of two separate approaches: (i) numerical integration of a stirred $n$-body pointvortex model with passively advected tracers, and (ii) analytic analysis of a model of randomly distributed vortices. In the $n$-body simulation, the flow is built from a collection of point vortices constrained to move in a plane. A stationary state is reached in which constant stirring is provided by discrete vortex creation at a fixed rate and dissipation occurs when oppositely signed vortices merge. The details of that model are given in Sec. II. The model is evolved numerically and in Sec. III probability density functions (PDFs) of the resulting Lagrangian velocity increments are presented. One of the advantages of the simple geometry considered is that approximations for these distributions can be derived analytically. This forms the basis of the second approach, bivariate transformation of random variable analysis of random distributions of vortices, presented in Sec. IV. The calculations reveal the dynamics underlying the point-vortex simulations and, perhaps, laboratory experiments. These connections are discussed in Sec. V.

\section{POINT-VORTEX MODEL}

We investigate a continuously stirred $n$-body point-vortex model. Point vortices are randomly generated with Gaussianly distributed intensity in a two-dimensional singly periodic domain. The domain has dimension of $x_{\max }^{2}$, with vortex interactions truncated at a separation distance of $x_{\max }$. The vortex intensity is defined by the circulation $\Gamma$, so that the velocity induced at distance $\mathbf{r}$ from a point vortex is

$$
\mathbf{u}(\mathbf{r})=\frac{\Gamma}{2 \pi r}(\hat{z} \times \hat{r}),
$$

where $\hat{z}$ is a unit vector perpendicular to the plane of motion. When $N$ such vortices are present in the domain, the velocity at any location $\mathbf{x}$ is computed as the sum of the contributions from each individual vortex,

$$
\mathbf{u}(\mathbf{x})=\sum_{k=1}^{N} \frac{\Gamma_{k}}{2 \pi\left|\mathbf{x}-\mathbf{x}_{k}\right|}\left[\hat{z} \times\left(\widehat{x-x_{k}}\right)\right] .
$$

The system is evolved with time, advecting each vortex by velocity induced by all the others at its location.

The merger of two vortices is imposed in the model whenever their separation is less than a fixed critical distance $r_{\text {min }}$. The new vortex position is taken as the amplitudeweighted average position, and the new intensity as the sum. The system would ultimately decay due to the merger of oppositely signed vortices except for an applied stirring by the injection of new point vortices at random locations in the 
domain. Injection occurs at a constant rate, with individual vortex intensities Gaussianly distributed with zero mean and standard deviation $\Gamma_{\text {rms. }}$.

The results presented in this paper are largely based on a simulation for which $2 \pi x_{\max }=64,2 \pi r_{\min }=1, \Gamma_{\mathrm{rms}} \approx 0.2$ (full width at half maximum $=0.5)$, and vortices are injected at a rate of 256 per unit time interval. Other model runs were computed, varying the injection rate of new vortices and the minimum separation distance allowed before the merging of vortices is enforced. While the simulations differ in detail, they share common statistical properties (the focus of this paper). The parameters chosen for the primary run presented are largely based on computational expedience, representing a compromise between the total number of vortices present in the domain and the total integration time achievable. As the parameters are defined, individual vortex velocities are normalized so that $u_{\theta}=\Gamma$ at a distance of $r_{\min }$, and a single Lagrangian orbit about a vortex of unit amplitude at that distance takes $2 \pi$ time units. The simulation was initiated with 32 randomly placed point vortices and evolved for 584 time units, reaching the statistically steady state illustrated by the snapshot Fig. 1(a). Figures 1(b) and 1(c) plot the evolution in number of vortices $N$ and the total circulation $\Gamma_{T}$ in the domain, while Fig. 1(d) plots the interaction energy of the $N$-vortex system,

$$
W=-\frac{1}{4 \pi} \sum_{\alpha}^{N} \sum_{\beta}^{N} \Gamma_{\alpha} \Gamma_{\beta} \ln \left(r_{\alpha \beta}\right),
$$

where the double sum extends over all vortex pairs $(\alpha \neq \beta)$ and $r_{\alpha \beta}$ is the distance between them.

In an infinite domain of constant point-vortex membership, $W$ is a constant of motion (e.g., [16]). Here, it shows significant variation with time. There are three reasons for this. Most importantly, the model processes of vortex creation and merger cause both energy dissipation and injection. Second, the domain periodicity, together with the limitation of maximum interaction distance, can cause discontinuous changes in the global vortex configuration as vortices shift location from one side of the domain to the other. Finally, the numerical integration scheme employed is weakly dissipative, particularly during close encounters of like signed vortices. Much more carefully conservative integration schemes have been developed (e.g., [17] and references therein), but are not explored here. In this paper we are primarily interested in the statistics of Lagrangian tracer motions within the point-vortex flow generated. For this, stirring of the flow by vortex creation is the most critical ingredient.

We note that, in this model, the merger of nearby vortices is imposed irrespective of vortex sign. For oppositely signed pairs this is readily interpreted as dissipation (although the interaction energy $W$ can increase or decrease depending on the consequent change in vortex configuration). Like sign mergers, particularly those that immediately follow vortex creation in the near vicinity of an already existing site, on the other hand, yield vortex amplification. While the flow remains strictly two-dimensional, these processes may to some degree mimic essential processes that are found in threedimensional turbulent flows but are generally absent from
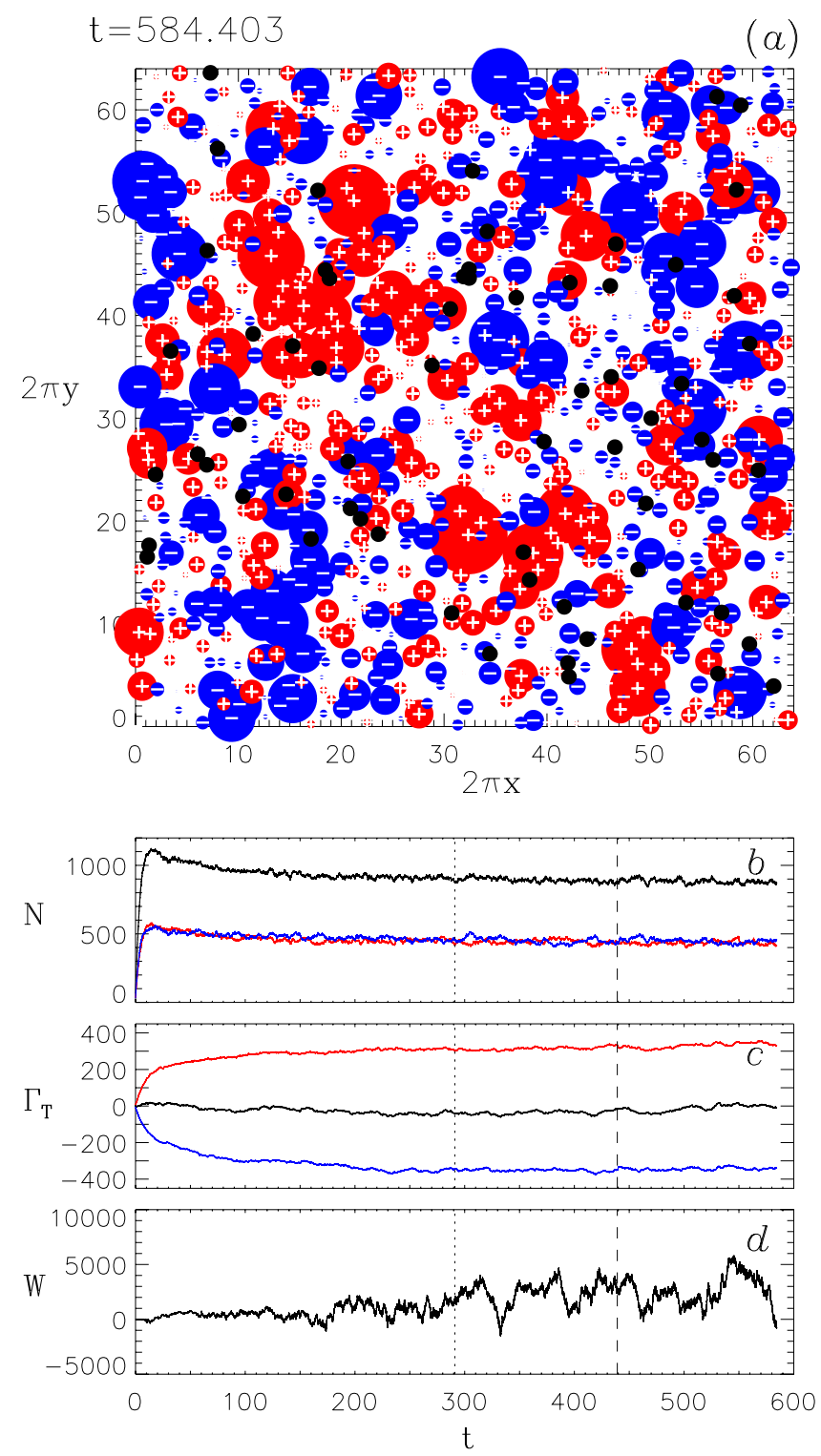

FIG. 1. (Color online) Top (a), instantaneous point-vortex distribution. Vortex locations are indicated by discs with area proportional to the vortex intensity. Blue (dark gray) symbols indicate negative (clockwise flow) and red (light gray) symbols indicate positive (counterclockwise flow) circulation respectively. Black symbols plot the positions of 64 passive Lagrangian tracer particles. Below, in (b) the number of vortices $N$, in (c) the total circulation of the vortices in the plane $\Gamma_{T}$, and in (d) the total vortex interaction energy $W$, as functions of time $t$. In (b) and (c), blue (dark gray) and red (light gray) traces plot values for negative and positive vortices separately. Spectral and statistical analyses in this paper examine data for time periods following the dotted and dashed vertical fiducial lines respectively.

two-dimensional models such as vortex tube reconnection and stretching, but that correspondence should not be over interpreted. Fundamentally the algorithm we have adopted introduces sources and sinks of vorticity otherwise unavailable to two-dimensional point-vortex systems. In the statistically steady state, energy injection equilibrates with dissipation. That state is achieved at late time in our simulation 


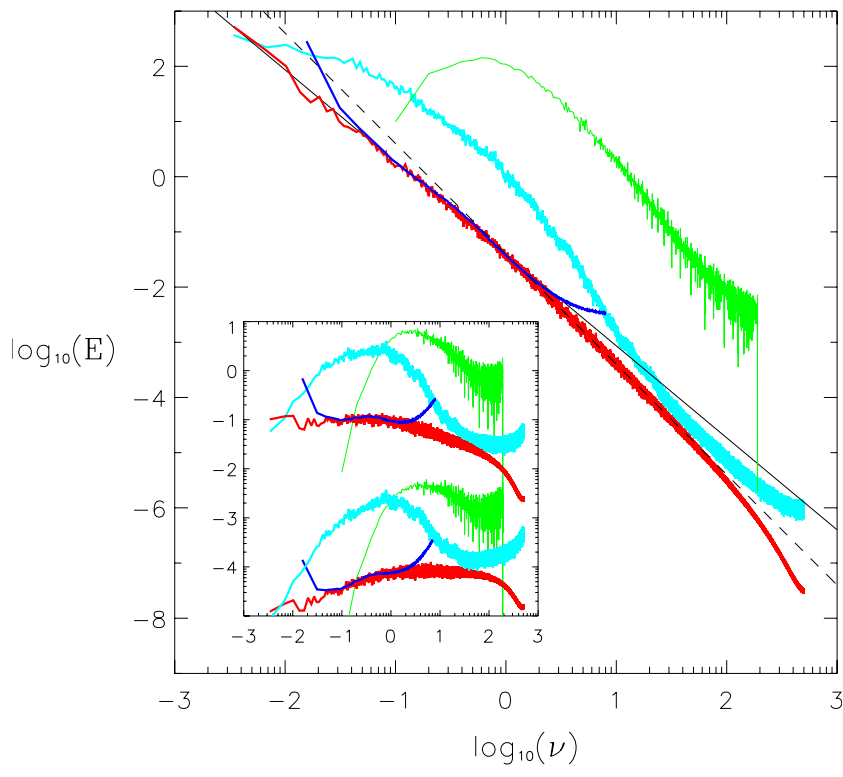

FIG. 2. (Color online) In red (dark gray), the Eulerian energy spectrum, $E(k)=k^{-1} u(k)^{2}$, determined by averaging the power spectra of the velocity time series taken at 64 Eulerian probe positions in the domain and imposing the Taylor hypothesis. Also, in dark blue (black), the average spatial power spectrum of the velocity reconstructed on a $1024^{2}$ spatial grid from 30 snapshots of the vortex positions. The light blue (light gray) curve plots the average velocity-power-spectrum of 64 Lagrangian tracer particles, with the green (medium gray) curve plotting the same quantity for laboratory data. The vertical positioning of the spectra is arbitrary. The solid line indicates Kolmogorov $k^{-5 / 3}$ scaling behavior, while the dashed line corresponds to a $k^{-2}$ scaling. Compensated spectra, by $k^{-5 / 3}$ (upper curves) and by $k^{-2}$ (lower curves), are shown in the inset. The Eulerian spectra show a significant $k^{-5 / 3}$ inertial range.

[Figs. 1(b)-1(d)], which shows nearly steady total vortex circulation and number, and strongly fluctuating but stationary values of $W$. Spectral and statistical analyses in this paper focus on the last 293.3 and 145.3 time units of the simulation, respectively (indicated by the dotted and dashed vertical fiducial lines in Figs. 1(b)-1(d)).

The model solutions share some similarities with forced turbulence. If one records the local velocity at fixed points in space (Eulerian probes), the energy spectra of the resulting time series (power spectrum of the velocity time series divided by the frequency to implement the Taylor hypothesis) display self-similar scaling with an exponent of $-5 / 3$ [red (dark gray) curves in Fig. 2]. This is supported by direct computation of the spatial energy spectrum via reconstruction of the two-dimensional velocity field over a discretized domain for 30 distinct realizations of the point-vortex positions [dark blue (black) curve in Fig. 2]. Such an inertial range spectrum is expected for three-dimensional turbulence [1] and has been observed in a wide variety of experimental situations [2]. In our model it extends for almost three decades before dropping to a value of -2 at higher frequencies. That higher frequency scaling likely reflects the random stirring of the domain, with the temporal power spectra of the total circulation [the time series illustrated by Fig. 1(c)] showing a $\nu^{-2}$ scaling at all frequencies. As we have stressed previously, although the vortices in this model are restricted to motions in a two-dimensional plane, some of the central ingredients of three-dimensional turbulence, such as vorticity production and amplification, have been introduced heuristically into the model by the vortex creation and merger processes. The usual conserved quantities of two-dimensional Navier-Stokes flows are not strictly preserved, and so one does not expect two-dimensional scaling laws to be observed.

Other properties of our model are not so simply related to turbulent fluid flows. For example, dissipation in the pointvortex model is achieved by a discontinuous merger process, so a flow Reynolds number is not easily defined. We may use the Eulerian spectrum to estimate the integral and Taylor microscales as

$$
L \propto \frac{\int[E(k) / k] d k}{\int E(k) d k}
$$

and

$$
\frac{1}{\lambda^{2}} \propto \frac{\int k^{2} E(k) d k}{\int E(k) d k}
$$

(e.g., [18]). This leads (to within factors of order unity) a value of $(L / \lambda)^{2} \approx 61000$. Using the relationships of homogeneous turbulence, that corresponds to a Taylor-based Reynolds number $R_{\lambda} \sim 250$, a value for which turbulence has been found to be fully developed in both direct numerical simulations and experimental studies [19,20], although some work suggests that perhaps a significantly higher value $\left(R_{\lambda}\right.$ $\sim 600-700)$ is needed to attain a robust Lagrangian inertial range [10]. Again, caution should be taken in making these comparisons.

\section{LAGRANGIAN MOTION AND STATISTICS: NUMERICAL SIMULATIONS}

Lagrangian tracers are dynamically advected in the model vortex flow; located at position $\mathbf{x}$ at time $t$, they assume the local fluid velocity and their position is incremented by $\delta \mathbf{x}$ $=\mathbf{u}(\mathbf{x}, t) \delta t$ during a time step of duration $\delta t$. Figure 3 plots two typical trajectories. These show swirling motions around vortices similar to those observed in resolved experimental tracking of Lagrangian tracers [4] and reminiscent of "trapping events" described in a recent three-dimensional simulations of fully developed homogeneous isotropic turbulence [12]. The tracer dynamics suggest that very strong accelerations are generated when the particles come into the immediate vicinity of vortices. This is supported by Fig. 3, in which the trajectory color reflects the magnitude of the particle acceleration at that point, and more quantitatively by Fig. 4 in which the $x$ components of the velocity and acceleration along a single Lagrangian trajectory are plotted, along with probability distributions of their values obtained 


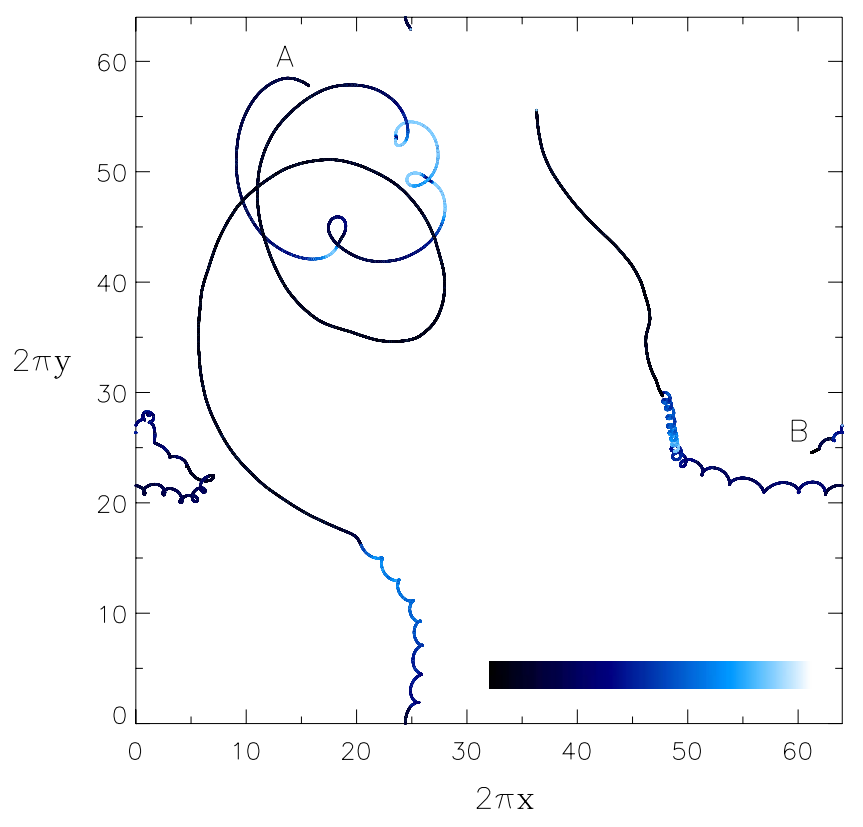

FIG. 3. (Color online) Two Lagrangian tracer trajectories spanning the last 35.6 time units of the simulation illustrated by Fig. 1 . Trajectories start at points labeled A and B, with color (gray scale) coding reflecting the magnitude of the velocity difference, $\sqrt{a_{x}^{2}+a_{y}^{2}}$, over temporal increment $\Delta t=0.001$ [the color table (gray scale) ranges in value from zero to 0.05 , higher values saturated]. Strong acceleration occurs during close encounters with point vortices. Trapping events [12] are quite common.

from 64 such trajectories. Lagrangian trajectories in the vicinity of a vortex show oscillations in velocity and large values of acceleration (the velocity difference between successive simulation time steps). The PDF of the Lagrangian velocity [Fig. 4(b)] is very close to Gaussian, as observed experimentally [5], while that of the acceleration is nonGaussian [Fig. 4(d)], significantly more so than that of a Gaussian random field [21].

A central question concerns how the velocity difference PDF changes with temporal increment, ranging from the flow turnover time to the smallest increment approximating the instantaneous particle acceleration. We define, for the velocity projected along the $x$ axis,

$$
a_{x}(t ; \tau) \equiv \Delta_{\tau} u_{x}(t)=u_{x}(t+\tau)-u_{x}(t)
$$

and examine the distribution of its value as a function of $\tau$. These PDFs are shown in Fig. 5. As in experimental measurements [5], one observes a continuous change in the distribution from Gaussian at large temporal increments (the velocity is then decorrelated) to highly non-Gaussian with extended tails for the smallest increments. The PDFs reflect dramatic changes in the acceleration time series as $\tau$ decreases [Figs. 5(b)-5(e)]. The time series display oscillations when the Lagrangian tracer orbits a vortex. The magnitude and frequency of the oscillations depend on both the period of the orbit and the temporal increment. As the temporal increment decreases, the frequency of the time series oscillations increases, reflecting those time periods when the Lagrangian tracer was in close proximity to a vortex. Lower
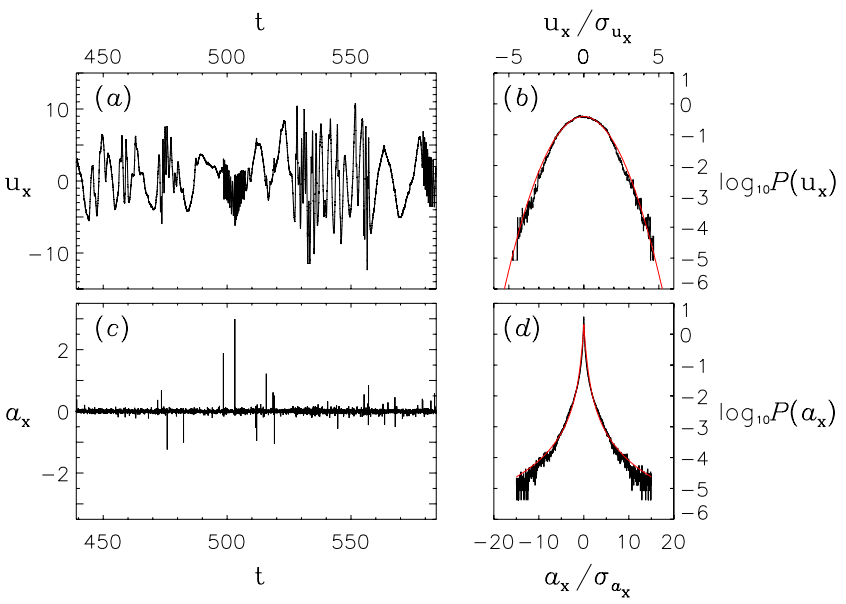

FIG. 4. (Color online) Time series, in (a) the Lagrangian velocity and in (c) the Lagrangian acceleration (velocity difference at smallest temporal increment available, the simulation time step $\tau$ $=0.001$ ). The last 35.6 time units plotted correspond to the Lagrangian trajectory A in Fig. 3, and the full time series are the last quarter of the simulation illustrated by Fig. 1. In (b) and (d), the velocity and acceleration PDFs obtained from the time histories of 64 such tracers. Values are normalized by their standard deviation $\left(\sigma_{u_{x}}\right.$ $=2.8$ and $\left.\sigma_{a_{x}}=0.018\right)$, and the total probability density integrates to unity. Red (gray) curves over plot in (b) a Gaussian fit to the velocity data and in (d) an analytic description of new vortex contributions to the Lagrangian acceleration (see Sec. IV).

frequency motions (more distant orbits), visible in time traces at larger temporal increment, yield accelerations close to zero at small temporal increment. This causes a decrease in the variance of the signal with decreasing $\tau$.

\section{VORTEX CONTRIBUTIONS: ANALYTICAL RESULTS}

Since the numerical model above (Sec. III) is based on simple point-vortex dynamics, the functional forms describing the dominant contributions to the velocity and acceleration (velocity-difference) distributions can be identified analytically. In this section we compare analytically derived PDFs to those obtained by randomly sampling randomly distributed vortices.

We consider first a single vortex having a fixed amplitude. We computed the velocity distribution it generates for points located at a fixed distance, and then for points distributed at random positions with respect to this single vortex. We subsequently consider a random collection of such vortices and compute the nearest neighbor and collective contributions. In comparing these results with those of the n-body model discussed previously, we are implicitly making the assumption that the statistics of many Lagrangian trajectories over long time periods are equivalent to those obtained from a random sampling of the vortex distribution at many positions. We base this ergodicity assumption on the random stirring of the $n$-body model flow, which stochastically alters both the vortex distribution in the domain and the spatial trajectories of the Lagrangian tracers. Moreover, our focus on acceleration over short temporal increments lessens the importance of 

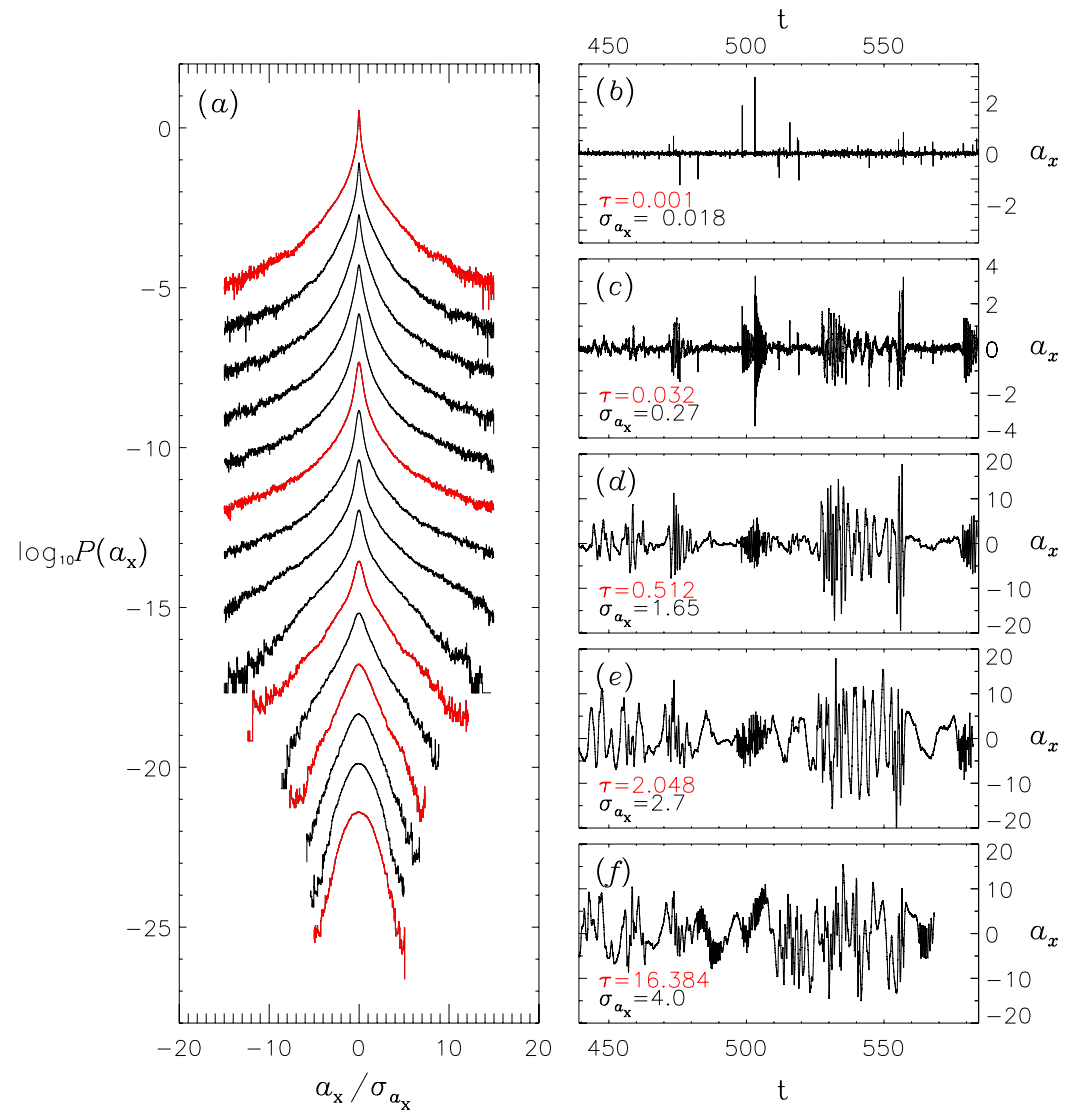

FIG. 5. (Color online) In (a), PDFs of the Lagrangian velocity difference (acceleration) as a function of temporal increment, with $\tau$ taking values from 0.001 to 16.384 , doubling for each curve from top to bottom. Using the velocity auto correlation function, one measures a Lagrangian integral time $T_{L} \sim 7.2$. Each successive distribution is offset vertically for clarity. Time traces on the right (b)-(e), are single Lagrangian particle contributions to the distributions plotted in red (gray) in (a), showing how the acceleration time series contributing to these distributions are sensitive to the temporal increment $\tau$. long time correlations. However, the assumption proves wrong. While we will show that the random stirring in the $n$-body model is critical to the velocity-difference PDFs at smallest temporal increment, the highly Gaussian Lagrangian velocity distribution strongly reflects trapping events in the flow, and cannot be explained on the basis of random sampling of the vortex field [13].

\section{A. Single point vortex}

In the plane perpendicular to the axis of a line vortex, the fluid velocity is $\mathbf{u}=A_{0} / r \hat{\theta}$, where $\hat{\theta}$ is the unit vector in the azimuthal direction, $r$ is the radial distance from the vortex site, and $A_{0}=\Gamma / 2 \pi$ [Eq. (1)]. The projection of this azimuthal velocity onto any direction in the plane, $x$ for example, is $u_{x}=\left(A_{0} / r\right) \sin \theta$, where $\theta$ is the angle between the position radius vector measured from the vortex site and the velocity projection direction. At a fixed radial distance $(r$ $=1$ ) the angular probability density $P(\theta)$ is uniform with $\theta$, and the probability density of the projected velocity is

$$
P\left(u_{x}\right) \propto \frac{1}{\cos \theta},
$$

where $\theta=\sin ^{-1}\left(u_{x} / A_{0}\right)$, so that

$$
P\left(u_{x}\right) \propto\left[1-\left(u_{x} / A_{0}\right)^{2}\right]^{-1 / 2} .
$$

The probability of observing any value of the projected velocity between 0 and $A_{0}$ is finite since $\int P\left(u_{x}\right) d u_{x}=\int A_{0} d \theta$, but the probability density diverges for $\theta= \pm \pi / 2$ corresponding to $u_{x}= \pm A_{0}$ where $d u_{x}$ goes to zero for a given $d \theta$ (Fig. 6). Note that this distribution not only describes the velocity of the simple point vortex sampled at constant radius, but also the projection onto a chosen direction of any randomly oriented vector uniformly distributed in direction and of constant magnitude. These very different physical interpretations cannot be distinguished based on PDFs alone. This is critical when assessing the importance of trapping events in the n-body flows (Sec. V A).

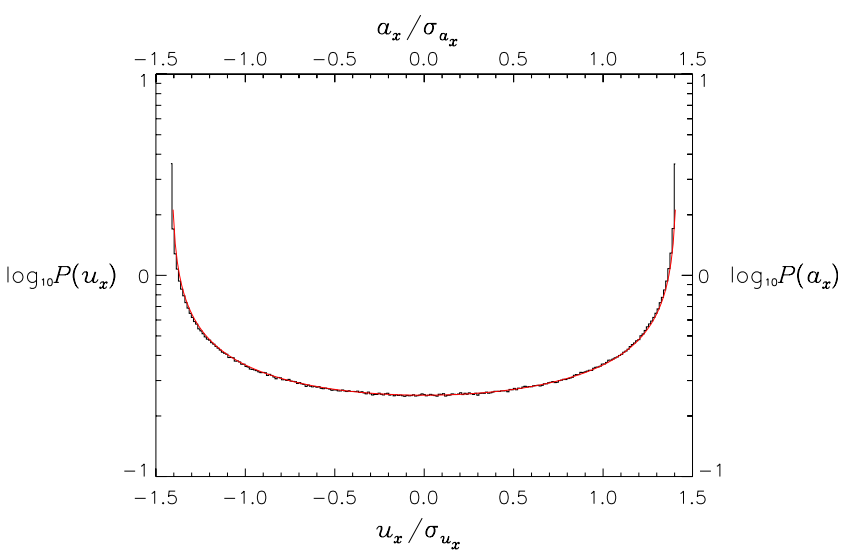

FIG. 6. (Color online) Logarithm of the probability densities of $u_{x}$ and $a_{x}$ for a point-vortex flow sampled uniformly in azimuthal position $\theta$ and at fixed radius $r$. Random sampling of a single point vortex at fixed distance is shown in black. The analytic functions describing these distributions [Eqs. (7) and (10) in text] are overplotted in red (gray). 
For this simple flow, sampled at constant radial distance, the projected Lagrangian velocity difference has the same distribution as the projected velocity itself. The velocity difference is

$$
\begin{aligned}
a_{x}= & A_{0} \sin (\theta+\delta)-A_{0} \sin \theta=A_{0}(\sin \theta \cos \delta+\cos \theta \sin \delta \\
& -\sin \theta),
\end{aligned}
$$

where $\delta=\omega \tau=A_{0} \tau$, with $\omega$ the angular velocity about the vortex site and $\tau$ the temporal increment between velocity measurements. The probability density of $a_{x}$ is

$$
P\left(a_{x}\right) \propto \frac{1}{\cos \theta \cos \delta-\sin \theta \sin \delta-\cos \theta},
$$

where $\theta$ is determined by inverting Eq. (9) for any given $a_{x}$ [22]. The velocity-difference variance is $\left\langle a_{x}^{2}\right\rangle \propto 1-\cos \delta$, and when scaled by the square root of the variance, all moments of the velocity-difference probability density are independent of the temporal increment $\tau$.

When the radial distance to the line vortex is not fixed, but instead the flow is sampled randomly in the plane perpendicular to its axis, $\theta$ is distributed uniformly, as before, but $P(r) \propto r$. By a bivariate transformation of the random variables (e.g., $[23,24]$ and this paper's Appendix A) the probability density for the projected velocity $u_{x}=A_{0} / r \sin \theta$ is then

$$
P\left(u_{x}\right) \propto \frac{1}{u_{x}^{3}},
$$

and, for small $\delta=A_{0} / r^{2} \tau, a_{x}=A_{0} \delta / r \cos \theta$ with probability density

$$
P\left(a_{x}\right) \propto \frac{1}{a_{x}^{5 / 3}} .
$$

The upper curves in Figs. 7(a) and 7(b) compare these analytical expressions with probability densities obtained by a random sampling of the point-vortex flow in a plane. The two match for $\left|u_{x}\right|>A_{0} / r_{\max }$ and $\left|a_{x}\right|>A_{0}^{2} \tau / r_{\text {max }}^{3}$. For values smaller than these, the probability density of the random sampling reflects the limited spatial extent of the domain, 0 $<r<r_{\max }$.

\section{B. Nearest neighbor contribution}

Consider next many equal amplitude point vortices randomly distributed in a plane with number density $n$. For any point in the plane, the probability density of the nearest neighbor distance is $P(r)=2 \pi n r e^{-\pi n r^{2}}$ (Appendix B, [25]). Taking the velocity to be locally only that contributed by the nearest neighbor vortex, transformation of random variables yields

$$
P\left(u_{x}\right)=\frac{\pi n}{u_{x}^{3}} e^{-\pi n /\left(2 u_{x}^{2}\right)}\left[I_{0}\left(\frac{\pi n}{2 u_{x}^{2}}\right)-I_{1}\left(\frac{\pi n}{2 u_{x}^{2}}\right)\right]
$$

[lower red (gray) curve in Fig. 7(a)] where $I_{0}$ and $I_{1}$ are modified Bessel functions of integer order [26]. Similarly, $P\left(a_{x}\right)$ can be written analytically in terms of generalized hy-

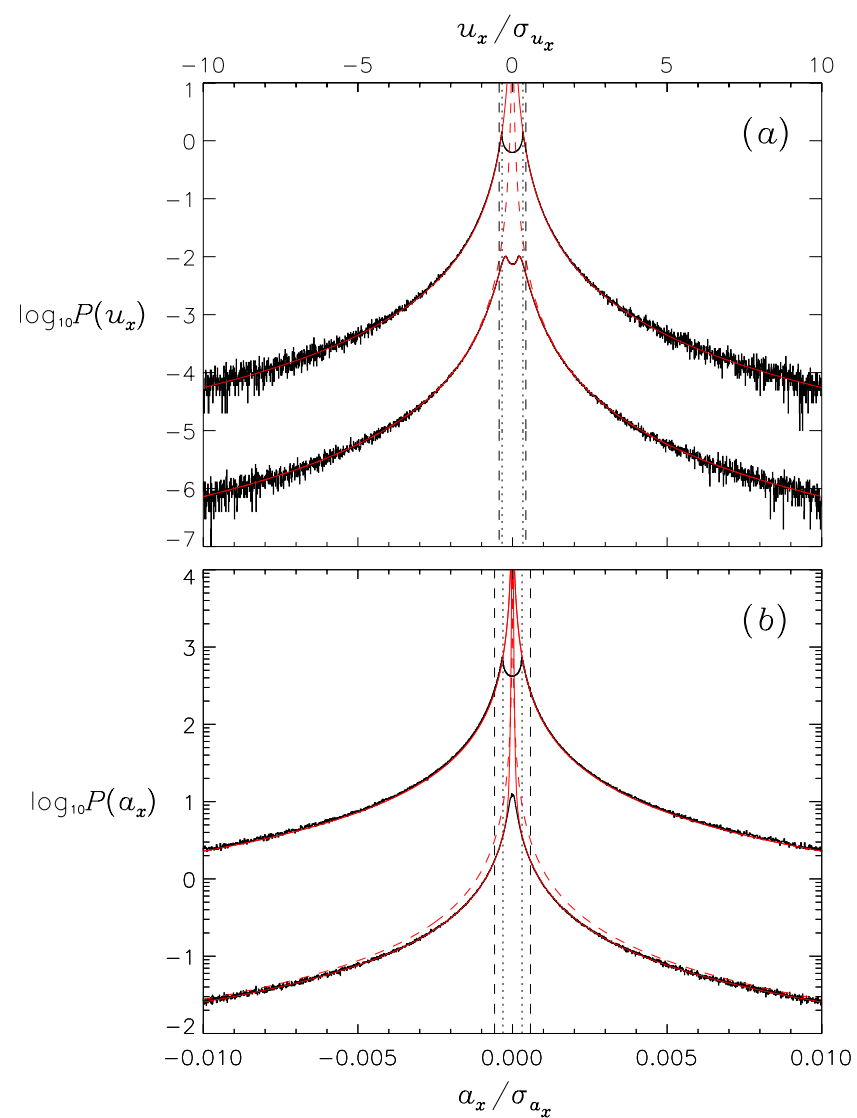

FIG. 7. (Color online) Upper curves in both (a) and (b): Logarithm of the probability densities of $u_{x}$ and $a_{x}$ for a single pointvortex flow sampled randomly in the $x_{\text {max }}^{2}$ plane. The analytic functions describing these distributions [Eqs. (11) and (12) in text] are overplotted in red (gray). The limits, $\left|u_{x}\right|=A_{0} / r_{\max }$ and $\left|a_{x}\right|$ $=A_{0}^{2} \tau / r_{\max }^{3}$, beyond which the random sampling contributions are independent of domain size, are indicated with vertical dotted fiducial lines. For clarity, only the very core of the velocity difference $a_{x}$ distribution is shown. Lower curves in both (a) and (b): Logarithm of the probability densities of the nearest neighbor contributions to $u_{x}$ and $a_{x}$ for a collection of identical amplitude point vortices randomly distributed in a plane and sampled randomly in the same two-dimensional space. The analytic functions describing these distributions [Eqs. (13) and (14) in text] are over-plotted in red (gray). Vertical dashed fiducial lines indicate where $\left|u_{x}\right|$ $=A_{0} / r_{m}$ and $\left|a_{x}\right|=A_{0}^{2} \tau / r_{m}^{3}$, where $r_{m}=0.5 / \sqrt{n}$ is the mean vortex spacing. Dashed red (gray) curves plot the lowest order power law contributions to the distributions [Eqs. (11) and (12)]. Only in the very core do these differ from the complete function, even over the very restricted domain shown in (b).

pergeometric functions, but perhaps more usefully as

$$
P\left(a_{x}\right)=n \frac{\tau^{2 / 3}}{a_{x}^{5 / 3}}\left[a_{0}+a_{1} n\left(\frac{\tau}{a_{x}}\right)^{2 / 3}+a_{2} n^{2}\left(\frac{\tau}{a_{x}}\right)^{4 / 3}+\cdots\right]
$$

[lower red (gray) curve in Fig. 7(b)] where the numerical coefficients $a_{n}$ can be evaluated analytically.

Monte Carlo sampling (random sampling of randomly generated vortex distributions) of 1500 identical stationary 


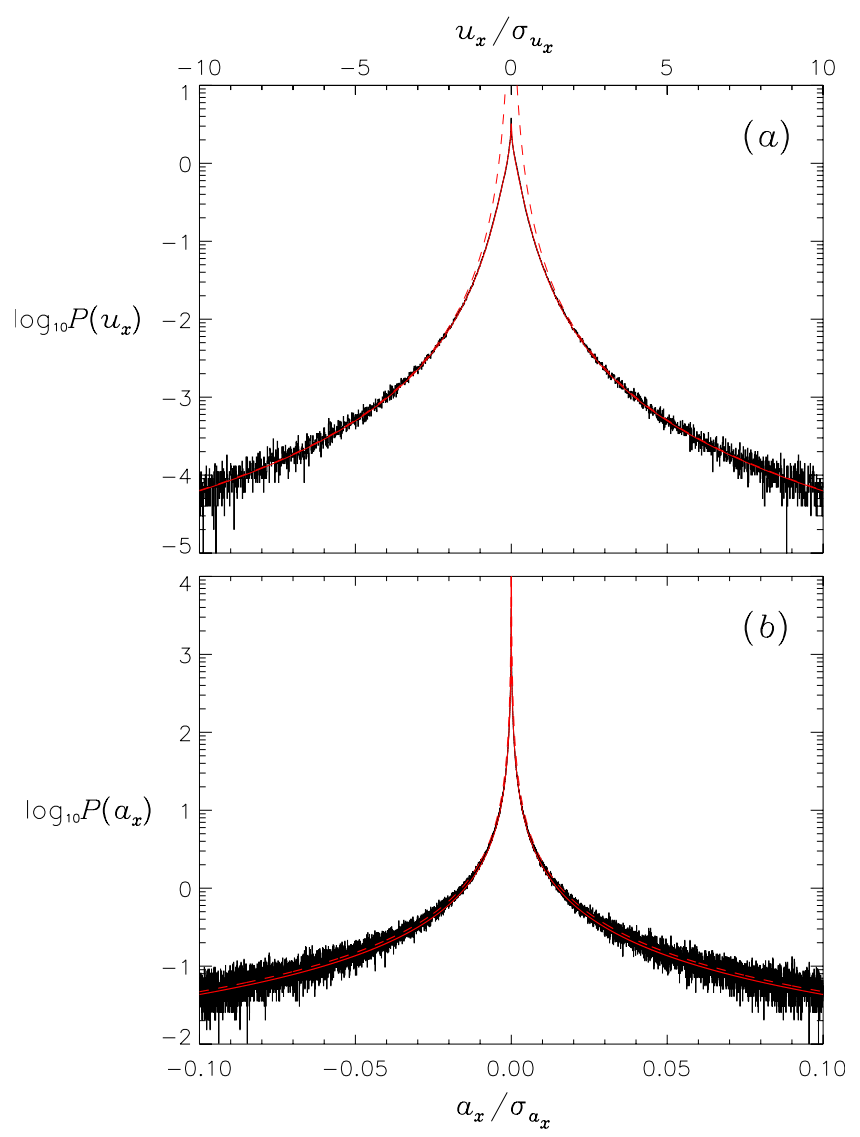

FIG. 8. (Color online) Logarithm of the probability densities of the nearest neighbor contributions to $u_{x}$ and $a_{x}$ for a collection of point vortices randomly distributed in a plane, with Gaussianly distributed amplitudes, and sampled randomly in the same twodimensional space. The analytic functions describing these distributions [Eqs. (15) and (16) in text] are overplotted in red (gray). Dashed red (gray) curves plot the lowest order power law contributions to the distributions [barely distinguishable from the complete function in (b), even over the very restricted domain shown].

vortices randomly distributed in an $x_{\max }^{2}$ domain with Lagrangian tracer advection over increment $\tau=0.001$ yields the distributions plotted in black as the lower curves in Fig. 7. Only in the core of these distributions, where $\left|u_{x}\right| \lesssim A_{0} / r_{m}$ and $\left|a_{x}\right| \lesssim A_{0}^{2} \tau / r_{m}^{3}$, with $r_{m}=0.5 / \sqrt{n}$ the mean vortex spacing, are the higher order terms in the analytic expressions significant. In the wings, the distributions are accurately described by the lowest order terms, equivalent to the power law expressions of Eqs. (11) and (12).

The same is true when the vortex amplitudes are Gaussianly distributed rather than of constant amplitude (Fig. 8). In this case the distributions are given analytically by

$$
\begin{aligned}
P\left(u_{x}\right)= & \frac{2}{\pi^{3}} \sqrt{\frac{1}{u_{x}^{2}+2 \pi n \sigma^{2}}}\left[K\left(\frac{2 \pi n \sigma^{2}}{u_{x}^{2}+2 \pi n \sigma^{2}}\right)\right. \\
& \left.-E\left(\frac{2 \pi n \sigma^{2}}{u_{x}^{2}+2 \pi n \sigma^{2}}\right)\right],
\end{aligned}
$$

where $K$ and $E$ are elliptical integrals of the first and second kind $[26]$ and $\sigma^{2}$ is the amplitude variance, and

$$
P\left(a_{x}\right)=n \frac{\left(\sigma^{2} \tau\right)^{2 / 3}}{a_{x}^{5 / 3}}\left[a_{0}+a_{1} n\left(\frac{\sigma^{2} \tau}{a_{x}}\right)^{2 / 3}+a_{2} n^{2}\left(\frac{\sigma^{2} \tau}{a_{x}}\right)^{4 / 3}+\cdots\right]
$$

where the $a_{n}$ are analytic numerical constants [different from those in Eq. (14)]. We emphasize that, given the point vortex density, amplitude variance, and temporal increment, the randomly sampled nearest neighbor contributions to the velocity and acceleration PDFs can be calculated exactly.

\section{Collective contributions}

Finally, we relax the nearest neighbor constraint and consider the velocity and velocity difference observed at a given location due to many point vortices distributed randomly in a plane and interacting through mutual advection.

Since the velocity at any observation point is the sum of those contributed by each individual vortex, and since the individual vortex contributions have power law distributions, the central limit theorem suggests that the resulting projected velocity distribution at any given observation point should be Gaussian in its core [27]. This is confirmed by randomly placing $N$ point vortices with Gaussianly distributed amplitudes in a domain and summing their contributions to the velocity measured at randomly located observation sites. Over many realizations of the vortex spatial distribution, the probability density of the projected velocity is Gaussianly distributed at low velocities and follows the nearest neighbor distribution previously derived [Eq. (15) in the wings (Fig. 9)]. The Gaussian core widens as the number density of vortices increases, but there is only slow convergence to Gaussianity [28].

The collective velocity difference measured at given point is distributed quite differently. To measure this we let the observation point and the field vortex positions above evolve over temporal increment $\tau$ as in the full $n$-body simulation described in Sec. II, but with two fundamental differences: no creation or merger of vortices occurs, and both the observation site and the vortex amplitudes and positions are chosen randomly anew before each measurement, eliminating dynamics induced spatial and temporal correlations. Thus the measured velocity differences represents the purely advective contribution of a random collection of vortices over a given temporal increment. This velocity difference can be written

$$
u_{T}\left(x^{\prime}, t^{\prime}\right)-u_{T}(x, t)=\sum_{i}\left[u_{i}\left(x_{i}^{\prime} ; x^{\prime}, t^{\prime}\right)-u_{i}\left(x_{i} ; x, t\right)\right],
$$

where $u_{T}(x, t)=\sum_{i} u_{i}\left(x_{i} ; x, t\right)$ is the total velocity at position $x$ and time $t$ contributed by vortices located at $x_{i}$. For a fixed Eulerian probe the observation positions $x$ and $x^{\prime}$ are the same, but they differ for a Lagrangian tracer advected with the flow. For neither type of observation does the centrallimit theorem hold. For a Lagrangian tracer $x^{\prime}=x+u_{T} \tau$. This is not equal to $x+u_{i} \tau$ (advection by each vortex individually) and so the sum in Eq. (17) cannot be written as one over individual vortex contributions. Moreover, even for an Eulerian probe, $u_{i}\left(x_{i} ; x, t\right)$ depends on the relative position of the observation site at $x$ and the $i^{\text {th }}$ vortex at $x_{i}$. Since the indi- 

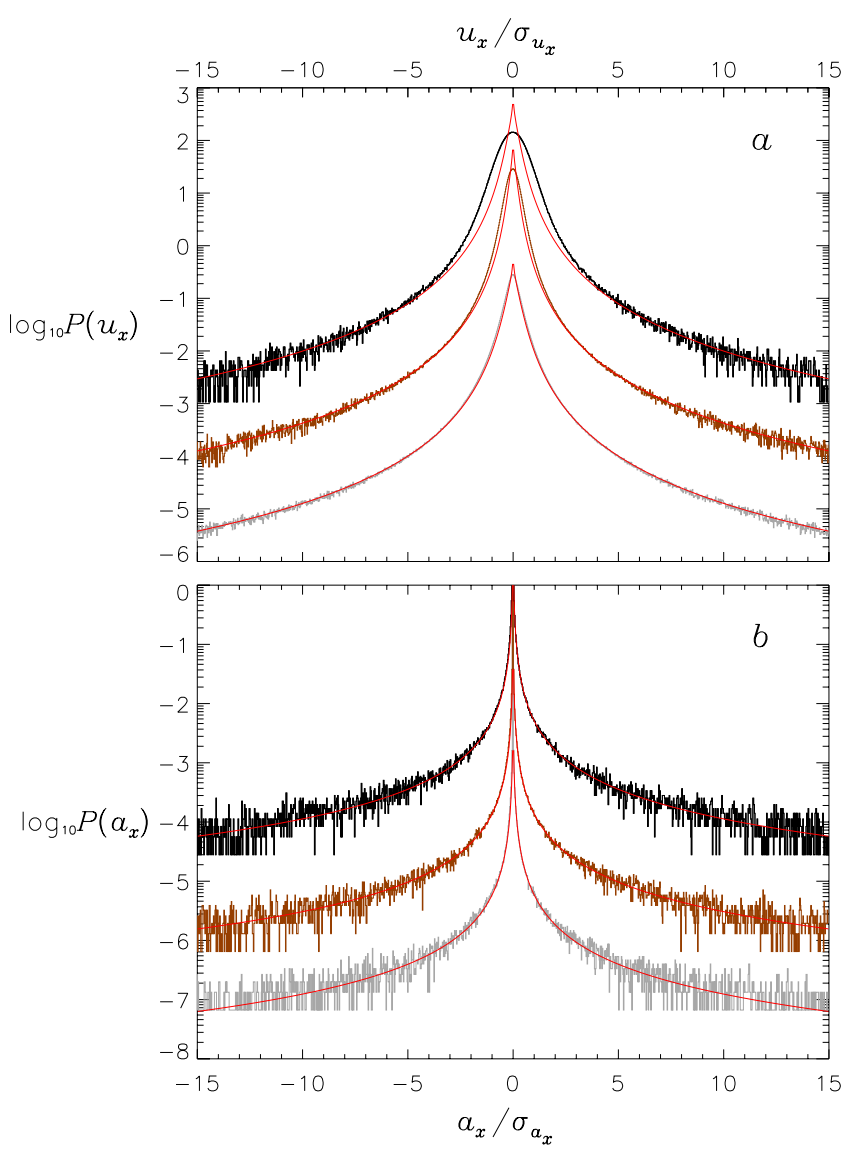

FIG. 9. (Color online) PDFs of the Lagrangian velocity (a) and acceleration (b) measured by randomly sampling many realizations of a random distribution of vortices with Gaussianly distributed amplitudes. The domain is identical to the full point-vortex simulation discussed in Sec. II and the velocity difference is calculated with temporal increment equal to the time step of that simulation. Curves are offset vertically for clarity with the vortex densities in the domain changing by two orders of magnitude from bottom to top (10 vortices, gray (bottom), 100 vortices, brown (middle), and 1000 vortices, black (top), in the domain respectively). Red (gray) curves plot the analytic nearest neighbor PDFs for each vortex density [Eq. (15) for $P\left(u_{x}\right)$ and Eq. (16) for $P\left(a_{x}\right)$ ] and match the distributions outside the core regions, where collective contributions at high number density can be important to $P\left(u_{x}\right)$ (see text).

vidual vortex motions depend on all other vortices, $x_{i}^{\prime}=x_{i}$ $+\sum_{j \neq i} u_{j}\left(x_{j} ; x_{i}, t\right) \tau$, the velocity-difference sum again cannot be expressed as a sum of individual and independent contributions. As a consequence, both the Eulerian and Lagrangian velocity difference are non-Gaussianly distributed even in the core [Fig. 9(b)].

As further evidence we have computed PDFs for the velocity difference under three scenarios [29]: Lagrangian tracers in a fixed (random) vortex field, fixed Eulerian tracers in an evolving vortex field, and Lagrangian tracers in an evolving vortex field. The distributions in all cases, the last illustrated by Fig. 9, show strongly non-Gaussian distributions with divergent cores and are well described by nearest neighbor contributions [Eq. (16)]. Recall that in this section the vortex system is not forced into a steady state via the creation and annihilation of vortices, as it was in the $n$-body model of Sections II and III. The vortex distributions here are randomly chosen anew for each measurement, so that "Lagrangian" and "Eulerian" refer to the method of sampling of the vortex configurations rather than to their dynamical evolution. The plots in Fig. 9 refer only to a random sampling of $N$ vortices of Gaussianly distributed amplitude.

\section{OBSERVATIONAL CONNECTION AND PHYSICAL INTERPRETATION}

\section{A. The importance of trapping events}

As discussed above, Monte Carlo sampling of the Lagrangian velocity and velocity-difference in random vortex fields yields distributions (Fig. 9) dominated by nearest neighbor scaling in the wings, with deviations only in the very core, and these only for the velocity distribution when the number density of point vortices is very high. This does not match the Lagrangian statistics of either our point-vortex simulation [Fig. 4(b)] or that of laboratory measurements [11]. In those, the Lagrangian velocity PDF is strictly Gaussian with no evidence for broader wings, even for values of velocity outside the expected Gaussian core. One might guess that this is due to the assumed Gaussian distribution of the vortex amplitudes used in the Monte Carlo examples, but that is not the case. While the actual amplitude distribution of the vortices in the $n$-body simulation is not Gaussian, when this is accounted for in derivation of the randomly sampled Lagrangian PDFs only a small change to the core of the distribution results. Moreover, an Eulerian measurement of the velocity in the point-vortex model [made at fixed probe locations, brown (gray) curve in Fig. 10(a)] shows the distribution expected, Gaussian in the core with elevated nearest neighbor wings [30].

The key to the strict Gaussianity of the observed Lagrangian velocity probability distribution function lies in the dynamics of the Lagrangian tracers. The Lagrangian trajectories do not sample the vortex field randomly, but instead undergo a series of trapping events during each of which they sample the vortices at a nearly constant radius, and that radius depends on the amplitude of the trapping vortex. This suggests that in the derivation of $P\left(u_{x}=A_{0} / r \sin \theta\right)$ it is incorrect to treat $A_{0}$ and $r$ as independent random variables. The vortex circulation and the distance at which a Lagrangian tracer is trapped are related; trapping by a vortex of low amplitude can occur only at very close distance. If one takes velocities of trapped Lagrangian particles $U_{0}=A_{0} / r$ to be distributed as

$$
P\left(U_{0}\right)=\frac{1}{2 \sigma^{2}}\left|U_{0}\right| \exp \left(\frac{-U_{0}^{2}}{2 \sigma^{2}}\right),
$$

then the probability density of $P\left(u_{x}\right)$ is exactly Gaussian, as observed. The curious implication of this result is that the Lagrangian trajectories may be composed of a series of trapping events each of well defined and coherent motion, but the velocity amplitudes of those events are distributed in such a way [Eq. (18)] to produce a strictly Gaussian velocity distribution when many such events are included in the time series analyzed. The probability density function then shows 


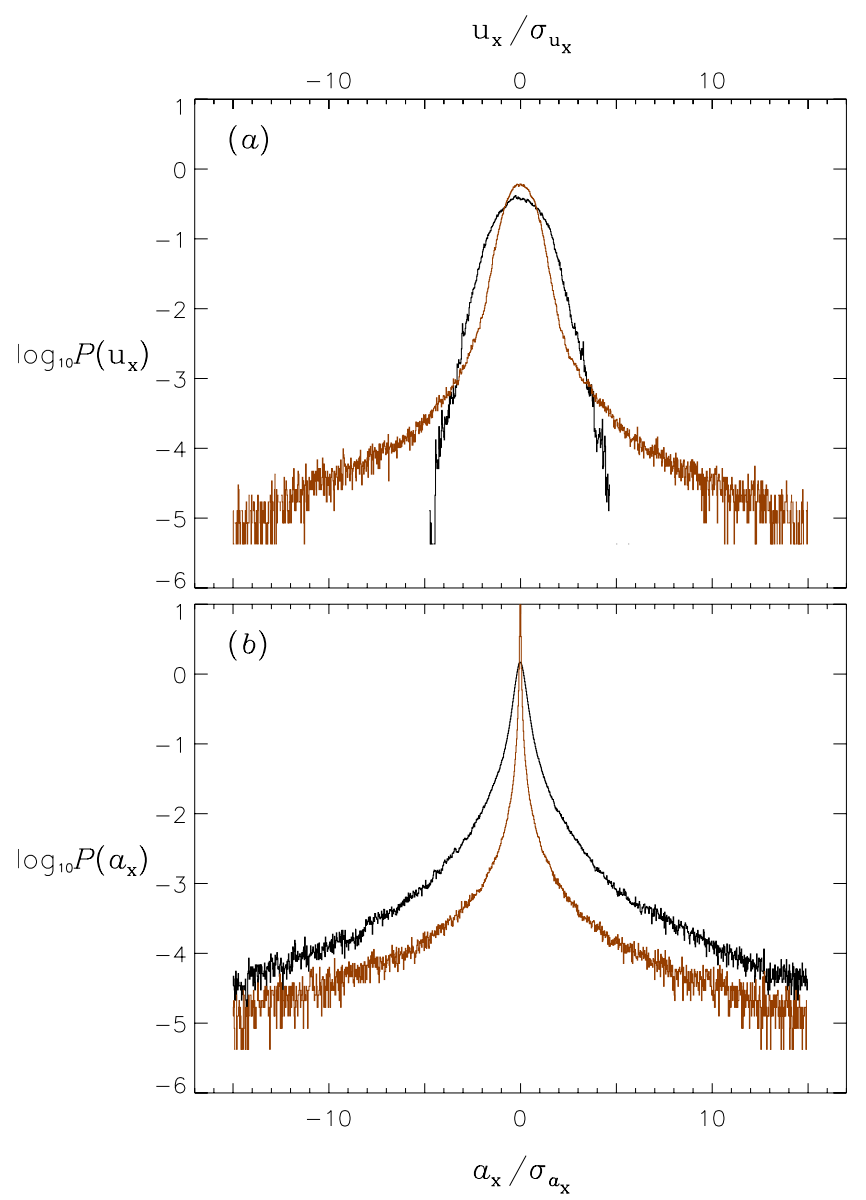

FIG. 10. (Color online) The Lagrangian (black) and Eulerian [brown (gray)] velocity (a) and velocity-difference (b) probability density functions from the stirred point-vortex model. A temporal increment of $\tau=0.032$, corresponding to the viscous time scale as estimated from the Lagrangian autocorrelation function and the flow Reynolds number, was used. The Eulerian probe measures distributions in agreement with random nearest neighbor sampling of the point-vortex field. The Lagrangian tracers do not.

no hint of the power law wings that dominate a random sampling of the same vortical flow field. Alternatively, independent normally distributed velocity components in two dimensions also lead to the two-dimensional Maxwellian speed distribution [Eq. (18)], suggesting short correlation times for the Lagrangian velocity vector orientation [31]. This ambiguity between vortical and randomly oriented motions, previously noted in Sec. III A when discussing single pointvortex contributions at fixed radius, cannot be resolved by the PDF alone.

The velocity-difference PDFs are more difficult to interpret. For the laboratory data they are limited by the minimum temporal increment achievable. For the point-vortex simulations, where arbitrarily small temporal increments can be achieved, they are complicated by the contributions from new vortex creation.

\section{B. The Lagrangian acceleration}

Let us first stress that the point-vortex analytical models in Sec. IV all show velocity-difference PDFs with significant

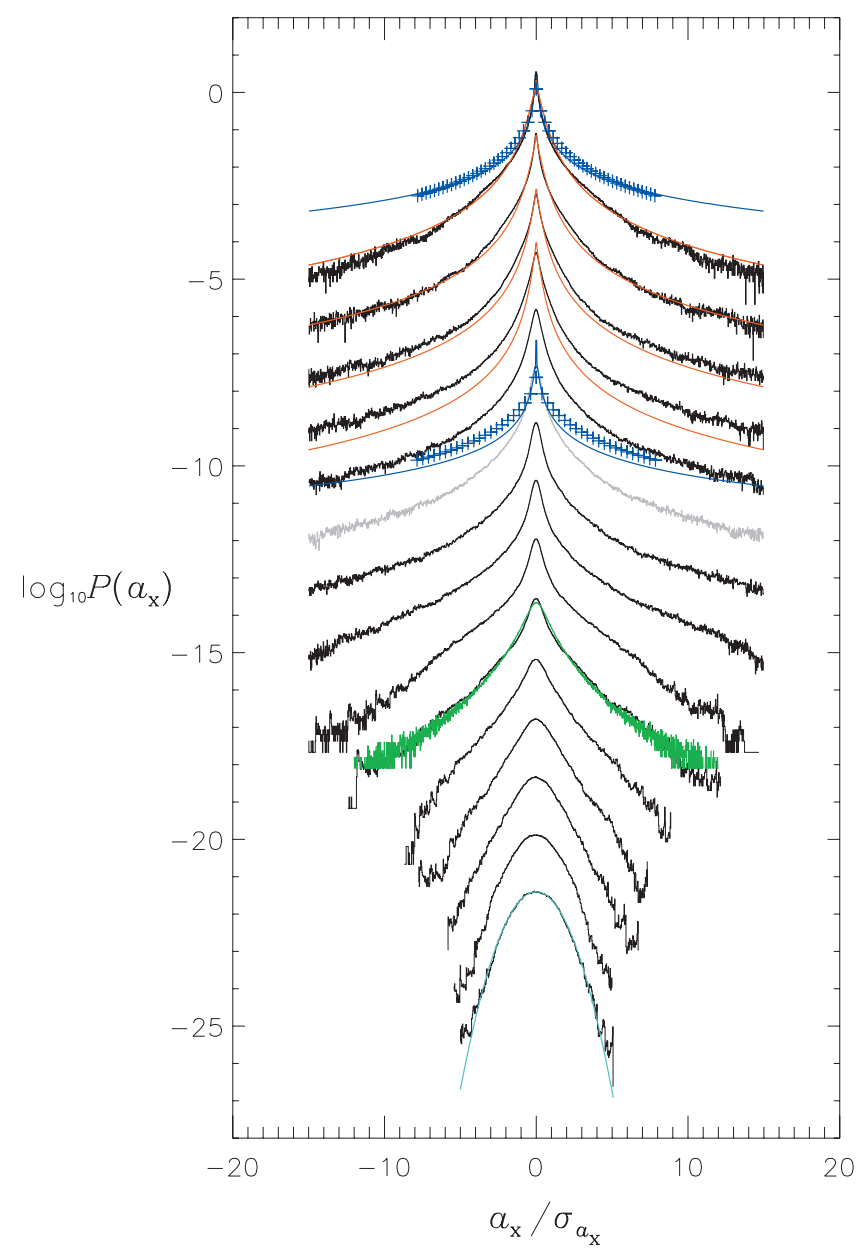

FIG. 11. (Color) PDFs of Lagrangian acceleration for the pointvortex flow simulation (black curves), and experimental data (green curve from [5]). Overplotted are statistical models of the distributions: cyan, Gaussian; blue curve, nearest neighbor acceleration [Eq. (16)], blue crosses, trapping event distribution, and red curves new vortex velocity distribution. These were calculated from input parameters of the model and normalized in amplitude. Temporal increment $\tau$ takes values from 0.001 to 16.384 , doubling for each curve from top to bottom. Each successive distribution is offset vertically for clarity. Gray curve plots the distribution for increment equal to the estimated viscous time scale in the simulation.

insensitivity to details of the vortex amplitude distributions, sampling of the field, or collective contributions, outside of the resulting distribution core, and that core region narrows with decreasing temporal increment. Outside of the narrow core, the velocity-difference distributions closely approximate the dominant $P\left(a_{x}\right) \propto a_{x}^{-5 / 3}$ nearest neighbor contribution in all cases, even when the contributions from many vortices in the domain are considered (Fig. 9).

In hydrodynamic experiments, both the stirred twodimensional (2D) point vortex flows presented here and laboratory measurements, the situation is quite different. Plotted in Fig. 11 are the PDFs of Lagrangian acceleration $\Delta_{\tau} u_{x}$ for the point-vortex simulation as a function of temporal increment $\tau$ (black curves), with $\tau=\tau_{0} 2^{n}, n$ ranging from 0 to 14 , top to bottom, and $\tau_{0}=0.001$, the time step of the simulation. At large temporal increments the velocity- 
difference PDF coincides with that of the velocity itself, and is described by a Gaussian distribution (cyan curve in Fig. 11). As the increment scale is reduced the PDFs evolve from Gaussian to having stretched exponential tails. This is in good agreement with 3D turbulent flow measurements at the smallest temporal scales investigated in experiments (green curve, from [5]). At yet smaller temporal increment, the Lagrangian velocity difference in our point-vortex flow becomes increasingly intermittent. The gray curve in Fig. 11 plots the distribution for increment equal to the estimated viscous time scale in the simulation. Note, however, that the distribution does not approach that expected from a nearest neighbor random (overplotted in blue in Fig. 11) or trapped (blue crosses) sampling of a Monte Carlo vortex field. The latter trapped sampling distribution can be computed analytically in terms of the Meijer $G$ functions [32] by making use of the trapping distribution [Eq. (18)], the width of the velocity distribution, the vortex field amplitude variance $\Gamma_{\text {rms }}$, and the temporal increment.

Thus we find that neither the nearest neighbor nor the trapped orbit Lagrangian velocity-difference PDF corresponds to that observed at short temporal increments in laboratory experiments or our $n$-body point-vortex model. This is true both for temporal increment of order the viscous time scale (gray curve) and one much smaller (of order the simulation time step, upper most curve in Fig. 11). The latter increment is likely not realistic, since vortices merge and are create instantaneously in the simulation, but suggests the distribution trend. Our observation is that, as the temporal increment decreases, the velocity-difference PDF approaches the velocity PDF of the randomly sampled nearest new neighbor (overplotted in red in Fig. 11). This can be computed exactly, evaluating Eq. (15) using the number density and amplitude distribution width $\Gamma_{\text {rms }}$ of new vortices generated in the domain over the time period $\tau$. Over very small temporal increment, the only contribution to the velocity difference is from vortex reconfiguration, via creation in the model or flow instabilities in a real flow. At very small $\tau$, contributions from the motions of the Lagrangian tracer and the background vortices are negligible compared to changes induced by vortex reconfiguration.

\section{Discussion}

We have constructed a stirred two-dimensional pointvortex model which shares some properties with fully developed three-dimensional turbulence. While limited, the model has the advantage of allowing exact calculation of some possible contributions to the dynamics of fluid particles.

We find that the Gaussianity of the velocity distribution results from the particular nature of Lagrangian dynamics. Lagrangian particles follow specific trajectories. The tracers are successively trapped around vortices, and the exact Gaussianity of velocity distribution implies a specific statistical relationship between the distance of trapping and the intensity of the vortex, with the probability distribution of this ratio given by Eq. (18). Alternatively, the Lagrangian velocity vector may be undergoing random reorientation. These interpretations cannot be distinguished on the basis the PDF alone, may be critical in assessing the importance of trapping events at high Reynolds number [33], and remain a focus of future work.

Concerning the distribution of the Lagrangian velocity difference (acceleration), the velocity results suggest that it should also be dominated by nearest neighbor contributions during trapping. This is not the case. As the temporal increment is decreased toward the dissipation scale, the evolution of the PDF suggests (Fig. 11) that the limiting distribution results from vorticity reconfiguration within the entire flow volume. In the numerical model, this is due to the creation and merging of vortices within the domain. In a real physical flow this would reflect flow instability and dissipation.

Many studies have pointed out the physical importance of concentrated vortices to the dynamics and statistics of Lagrangian tracers. The sensitivity at small scales to global reconfiguration of the vorticity field implied by our model suggests that less local influences may also be important to measurements made at short temporal increments. This is in agreement with the observation (e.g., [5]) that the Lagrangian acceleration is dominated by pressure gradients. As pressure solves a Poisson equation formally equivalent to that in electrostatics, with vorticity and strain playing the role of negative and positive charges $[34,35]$, it is extremely sensitive to changes in the overall "charge" configuration. As the temporal increment decreases, and consequently so too does the direct contribution of Lagrangian tracer motion, reconfiguration of existing and/or generation of new vortical structures within the domain plays a larger role.

\section{ACKNOWLEDGMENTS}

Special thanks to A. Alexakis, M. Bourgoin, S. Criscuoli, D. Gough, T. Holzer, N. Mordant, D. Nychka, R. Volk, and J. Weiss.

\section{APPENDIX A: BIVARIATE TRANSFORMATION OF RANDOM VARIABLES}

Let $x$ and $y$ be independent random variables with probability densities $P(x)$ and $P(y)$, respectively, and joint probability density $P_{x y}(x, y)=P(x) P(y)$. Let $u=f(x, y)$ and $v$ $=g(x, y)$ be functions of the random variables with inverses $x=h_{1}(u, v)$ and $y=h_{2}(u, v)$. (Note that typically only one of these functions is one for which the probability density is desired, while the other is a dummy function chosen judiciously so that it can be easily integrated out of the joint probability density leaving that of the required function.) The joint probability density of $u$ and $v$ is (e.g. $[23,24]$ )

$$
P_{u v}(u, v)=P_{x y}\left(h_{1}, h_{2}\right)\left|\begin{array}{ll}
\frac{\partial h_{1}}{\partial u} & \frac{\partial h_{1}}{\partial v} \\
\frac{\partial h_{2}}{\partial u} & \frac{\partial h_{2}}{\partial v}
\end{array}\right|,
$$

and the individual probability densities for $u$ and $v$ can be determined by integration of their joint probability density over the range of the other variable: 


$$
P(u)=\int P(u, v) d v \text { and } P(v)=\int P(u, v) d u .
$$

As an example, consider two Gaussianly distributed independent random variables each with a mean value of zero and a variance equal to one and with joint probability

$$
P_{x y}(x, y)=\frac{1}{2 \pi} e^{-\left(x^{2}+y^{2}\right) / 2} .
$$

To derive the probability density of their product, define two functions, $u=x y$ and $v=y$, and their inverses, $x=h_{1}(u, v)$ $=u / v$ and $y=h_{2}(u, v)=v$. The joint probability density of $u$ and $v$ is then

$$
P_{u v}(u, v)=\frac{1}{2 \pi}\left|\begin{array}{cc}
\frac{1}{v} & -\frac{u}{v^{2}} \\
0 & 1
\end{array}\right| e^{-\left(u^{2} / v^{2}+v^{2}\right) / 2}=\frac{1}{2 \pi v} e^{-\left(u^{2} / v^{2}+v^{2}\right) / 2} .
$$

Integrating over the range of dummy variable $v$ by imposing symmetry about $v=0$ yields

$$
P(u)=\frac{1}{\pi} K_{0}\left(\sqrt{u^{2}}\right),
$$

where $K_{0}$ is the lowest order modified Bessel function of the second kind [26]. The probability density has a logarithmic singularity at zero which contributes nothing to the probability integral.

\section{APPENDIX B: PROBABILITY DENSITY OF NEAREST NEIGHBOR DISTANCE}

This derivation of the probability density function of the nearest neighbor distance when sampling a random distribution of points in a plane,

$$
P(r)=2 \pi n r e^{-\pi n r^{2}},
$$

follows [25].

Distribute $N$ points randomly in a plane of area $A$. If $N$ $=1$, then at any location in the plane, the probability of finding the neighbor at a distance between $r$ and $r+d r$ is

$$
d P_{1}=\frac{2 \pi r}{A} d r .
$$

For $N=2$, the probability of the nearest neighbor point being at a distance between $r$ and $r+d r$ is

$$
d P_{2}=d P_{1}\left(\frac{A-\pi r^{2}}{A}\right)+d P_{1}\left(\frac{A-\pi r^{2}}{A}\right),
$$

where two terms each express the probability that one of the points is the nearest neighbor, lies between $r$ and $r+d r$, and the other lies farther away, at a distance greater than $r$ and so not within $\pi r^{2}$. Similarly, for $N=3$, the probability of the nearest neighbor point being at a distance between $r$ and $r$ $+d r$ is

$$
\begin{aligned}
d P_{3}= & d P_{1}\left(\frac{A-\pi r^{2}}{A}\right)\left(\frac{A-\pi r^{2}}{A}\right)+d P_{1}\left(\frac{A-\pi r^{2}}{A}\right)\left(\frac{A-\pi r^{2}}{A}\right) \\
& +d P_{1}\left(\frac{A-\pi r^{2}}{A}\right)\left(\frac{A-\pi r^{2}}{A}\right),
\end{aligned}
$$

where each term expresses the probability that one of the points is the nearest neighbor and the other two lie farther away. Generalizing to $N$ points yields

$$
d P_{N}=N d P_{1}\left(\frac{A-\pi r^{2}}{A}\right)^{N-1}=\frac{N}{A} 2 \pi r\left(\frac{A-\pi r^{2}}{A}\right)^{N-1} d r .
$$

Letting $n=N / A$, the number density, and $\mu=A / \pi r^{2}$, this in turn can be rewritten as

$$
d P_{N}=2 \pi n r\left[\left(1-\frac{1}{\mu}\right)^{-\mu}\right]^{-n \pi r^{2}}\left(1-\frac{1}{\mu}\right)^{-1} d r .
$$

In the limit $\mu \rightarrow \infty$, when the nearest neighbor distance is much smaller than the size of the domain, this simplifies to

$$
d P_{N}=2 \pi n r e^{-\pi n r^{2}} d r,
$$

with mean

$$
\langle r\rangle=\int_{0}^{\infty} 2 \pi n r^{2} e^{-\pi n r^{2}} d r=\frac{1}{2 \sqrt{n}} .
$$

[1] A. N. Kolmogorov, Dokl. Akad. Nauk SSSR 30, 301 (1941).

[2] U. Frisch, Turbulence (Cambridge University Press, Cambridge, England, 1995).

[3] G. A. Voth, K. Satyanarayan, and E. Bodenschatz, Phys. Fluids 10, 2268 (1998).

[4] A. LaPorta, G. A. Voth, A. M. Crawford, J. Alexander, and E. Bodenschatz, Nature (London) 409, 1017 (2001).

[5] N. Mordant, P. Metz, O. Michel, and J.-F. Pinton, Phys. Rev. Lett. 87, 214501 (2001).

[6] G. Falkovich, K. Gawedzki, and M. Vergassola, Rev. Mod. Phys. 73, 913 (2001)

[7] M. S. Borgas, Philos. Trans. R. Soc. London, Ser. A 342, 379
(1993).

[8] L. Chevillard, S. G. Roux, E. Lévêque, N. Mordant, J.-F. Pinton, and A. Arnéodo, Phys. Rev. Lett. 95, 064501 (2005).

[9] L. Biferale, G. Boffetta, A. Celani, B. J. Devenish, A. Lanotte, and F. Toschi, Phys. Rev. Lett. 93, 064502 (2004).

[10] P. K. Yeung, Annu. Rev. Fluid Mech. 34, 115 (2002).

[11] N. Mordant, E. Lévêque, and J.-F. Pinton, New J. Phys. 6, 116 (2004).

[12] L. Biferale, G. Boffetta, A. Celani, A. Lanotte, and F. Toschi, Phys. Fluids 17, 021701 (2005).

[13] M. Wilczek, F. Jenko, and R. Friedrich, Phys. Rev. E 77, 056301 (2008). 
[14] L. Onsager, Nuovo Cimento, Suppl. 6, 279 (1949).

[15] R. H. Kraichnan, Phys. Fluids 10, 1417 (1967).

[16] D. M. F. Chapman, J. Math. Phys. 19, 1988 (1978).

[17] A. San Miguel, Phys. Rev. E 74, 046706 (2006).

[18] J. O. Hinze, Turbulence (McGraw-Hill, New York, 1959).

[19] A. Arnéodo et al., Europhys. Lett. 34, 411 (1996).

[20] A. Arnéodo et al., Phys. Rev. Lett. 100, 254504 (2008).

[21] A. Tsinober, P. Vedula, and P. K. Yeung, Phys. Fluids 13, 1974 (2001).

[22] For small values of $\delta, a_{x} \approx A_{0} \delta \cos \theta$ with probability density $P\left(a_{x}\right) \propto \frac{1}{\delta \sin \theta}$, where $\theta=\cos ^{-1}\left[a_{x} /\left(A_{0} \delta\right)\right]$, so that $P\left(a_{x}\right) \propto\left[\delta^{2}\right.$ $\left.-\left(a_{x} / A_{0}\right)^{2}\right]^{-1 / 2}$.

[23] G. Casella and R. L. Berger, Statistical Inference (Duxbury Press, Pacific Grove, 1990).

[24] R. V. Hogg and E. A. Tanis, Probability and Statistical Inference (Pearson Prentice Hall, Upper Saddle River, 1993).

[25] A. Alexakis (private communication).

[26] M. Abramowitz and I. R. Stegun, Handbook of Mathematical Functions with Formulas, Graphs, and Mathematical Tables (U. S. Government Printing Office, Washington, DC, 1972).
[27] D. Sornette, Critical Phenomena in Natural Sciences, (Springer, Berlin, 2000).

[28] J. B. Weiss, A. Provenzale, and J. C. McWilliams, Phys. Fluids 10, 1929 (1998).

[29] E. Lévêque, L. Chevillard, J.-F. Pinton, S. Roux, A. Arnéodo, and N. Mordant, J. Turbul. 8, 1 (2007).

[30] The elevated wings are expected from the point-vortex analysis here; they are not seen in Eulerian laboratory measurements (e. g., [2]). This is likely due to the unrealistic point-vortex core profile.

[31] N. Mordant, J. Delour, E. Lévêque, A. Arnéodo, and J.-F. Pinton, Phys. Rev. Lett. 89, 254502 (2002).

[32] S. Wolfram, The Mathematica Book (Wolfram Media/ Cambridge University Press, Cambridge, England, 1996).

[33] P. K. Yeung, S. B. Pope, E. A. Kurth, and A. G. Lamorgese, J. Fluid Mech. 582, 399 (2007).

[34] S. Douady, Y. Couder, and M.-E. Brachet, Phys. Rev. Lett. 67, 983 (1991).

[35] A. La Porta, G. A. Voth, F. Moisy, and E. Bodenschatz, Phys. Fluids 12, 1485 (2000). 